1453

\section{FACTORS INFLUENCING LONG-TERM IMMUNITY AGAINST HEPATITIS B - THE ROLE OF NATURAL BOOSTERS}

M. Pokorska-Lis ${ }^{1,2}$, M. Marczynska ${ }^{1,2}$

${ }^{1}$ Department of Children's Infectious Diseases, Medical University of Warsaw, ${ }^{2}$ Regional Hospital of Infectious Diseases, Warszawa, Poland

Background: Duration of protection against hepatitis $\mathrm{B}$ afforded by vaccination remains unknown. Factors influencing long-term immunity are uncertain.

Aim was to analyze factors influencing immunity against hepatitis $B$, including natural boosters, in 10-12-year old children vaccinated in infancy.

Methods: In 130 children aged 10-12 years, immunized against hepatitis $B$ in infancy, exposure to risk factors for HBV infection (hospitalization, surgical and dental interventions, blood transfusion, HBV infection in family member, piercing) were analyzed. Humoral immunity (anti-HBs) and immune memory (anamnestic response to booster) were determined.

Results: Protective anti-HBs level was found in $102 / 130(78 \%)$ children, in $43 / 130(33 \%)$ titer was high (100-999 IU/I), in 16/130 (12\%) very high ( $\geq 1000$ IU/I). 28/130 (22\%) had unprotective level, in $9 / 130(7 \%)$ antibodies were undetectable. No statistically significant influence of age and gender on anti-HBs titer distribution $(p<0.28 ; p<0.35)$ nor possession of unprotective antibody level $(p<$ $0.329 ; p<0.297$ ) was found. Anamnestic response to booster was revealed in $12 / 16(75 \%)$ and was positively correlated with younger age $(p<0,03)$, female gender $(p<0.01)$ and detectable prebooster anti-HBs $(p<0.02)$. Dental interventions and surgery had significant influence on possession of very high anti-HBs titers $(p<0.02 ; p<0.05)$. Children who had blood transfusion in neonatal period, significantly more frequently had unprotective antibody levels $(p<0.01)$.

Conclusions: Immune memory is positively correlated with younger age, female gender and detectable anti-HBs. Natural boosters, especially dental and surgical interventions, may enhance the post-vaccination immunity. In children who received blood transfusion, immunization may be less effective.

\section{HEPATITIS B VACCINATION EFFECTIVENESS IN GREENLAND}

M.L. Børresen ${ }^{1}$, T.G. Krause ${ }^{2}$, K. Ladefoged ${ }^{3}$, H.S. Pedersen ${ }^{4}$, A. Koch ${ }^{1}$

${ }^{1}$ Department of Epidemiology Research, ${ }^{2}$ Epidemiological Department, Statens Serum Institut, Copenhagen S, Denmark, ${ }^{3}$ Department of Internal Medicine, ${ }^{4}$ Primary Health Care Center,

Dronning Ingrids Hospital, Nuuk, Greenland

Background and aims: Greenland is a hepatitis $B$ (HBV) high-endemic country, but the only preventive measure against $\mathrm{HBV}$ is routine $\mathrm{HBsAg}$ testing of pregnant women and HBV vaccination of children born to HBsAg positive mothers.

Methods: This national wide study evaluated the effectiveness of the Hepatitis B vaccination program in Greenland by examine the frequency of $\mathrm{HBsAg}$ testing during pregnancy of all live-births from 1992 to 2000. Secondly we followed-up children born to HBsAg positive mothers in Greenland in the period 1992 to 2007 and determined their vaccination- and HBV status.

Results: The survey revealed more challenges in the Greenlandic HBV vaccination program. Firstly, only $65 \%$ of all live births were preceded by HBV testing of the mother during pregnancy. Secondly in the follow up study of children born to HBsAg positive mothers, we found that $20 \%$ had received no vaccination at all, and that only $58 \%$ of the children had received three or four vaccinations. Thirdly, $3 \%$ of the children born to HBsAg positive mothers were immune and $3 \%$ were chronically infected. Fourthly, the HBsAb level of immunized children was unexpectedly low indicating non responders or an ineffective vaccine.

Conclusions: Based on the results in this study we therefore urgently recommend changing the HBV vaccination strategies in Greenland. $\mathrm{HB}$ vaccination should be included in the childhood vaccination program and the procedures in the general screening programme of pregnant women should be evaluated and there should be an attenuated awareness of atrisk children born to HBsAg positive mothers. 